Research article

Open Access

\title{
Soluble Fas ligand inhibits angiogenesis in rheumatoid arthritis
}

\author{
Wan-Uk Kim ${ }^{1}$, Seung-Ki Kwok', Kyung-Hee Hong ${ }^{1}$, Seung-Ah Yoo', Jin-Sun Kong', \\ Jongseon $\mathrm{Choe}^{2}$ and Chul-Soo Cho ${ }^{1}$
}

\begin{abstract}
1Division of Rheumatology, Department of Internal Medicine, School of Medicine, Catholic University of Korea, Seoul 137-701, Korea
2Department of Microbiology and Immunology, Kangwon National University College of Medicine, Chunchon, Kangwon 200-701, Korea
\end{abstract}

Corresponding author: Chul-Soo Cho, chocs@catholic.ac.kr

Received: 10 Oct 2006 Revisions requested: 13 Nov 2006 Revisions received: 12 Mar 2007 Accepted: 26 Apr 2007 Published: 26 Apr 2007

Arthritis Research \& Therapy 2007, 9:R42 (doi:10.1186/ar2181)

This article is online at: http://arthritis-research.com/content/9/2/R42

(c) 2007 Kim et al.; licensee BioMed Central Ltd.

This is an open access article distributed under the terms of the Creative Commons Attribution License (http://creativecommons.org/licenses/by/2.0), which permits unrestricted use, distribution, and reproduction in any medium, provided the original work is properly cited.

\begin{abstract}
The characteristics of rheumatoid arthritis (RA) pathology include the infiltration of inflammatory leukocytes, the proliferation of synovial cells, and the presence of extensive angiogenesis, referred to as rheumatoid pannus. Fas ligand is critical to the homeostatic regulation of the immune response, but its role in the angiogenic process of RA remains to be defined. In this study, we investigated whether soluble Fas ligand (sFasL) induces synoviocyte apoptosis and regulates angiogenesis of endothelial cells in RA. The levels of sFasL were elevated in the synovial fluids of RA patients when compared to those of osteoarthritis (OA) patients, and they correlated inversely with vascular endothelial growth factor ${ }_{165}\left(\mathrm{VEGF}_{165}\right)$ concentrations. sFasL, ranging from 10 to $100 \mathrm{ng} / \mathrm{ml}$, induced

the apoptosis of RA fibroblast-like synoviocytes (FLS) in vitro, and thereby decreased VEGF ${ }_{165}$ production. In addition, sFasL

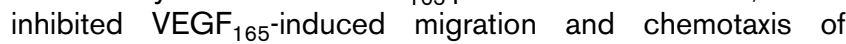
endothelial cells to basal levels in a manner independent of the Fas-mediated cell death. sFasL dose-dependently suppressed the VEGF $_{165}$-stimulated increase in pAkt expression in endothelial cells, which might be associated with its antimigratory effect on endothelial cells. Moreover, sFasL strongly inhibited neovascularization in the Matrigel plug in vivo. Our data suggest that sFasL shows anti-angiogenic activity within RA joints not only by inducing apoptosis of $V E_{165}$-producing cells but also by blocking VEGF 165 -induced migration of endothelial cells, independent of Fas-mediated apoptosis.
\end{abstract}

\section{Introduction}

Rheumatoid arthritis (RA) is a multi-systemic autoimmune disease of unknown etiology that is characterized by hyperplastic synovial membrane capable of destroying adjacent articular cartilage and bone $[1,2]$. The pathology of RA synovial membrane includes infiltration of inflammatory leukocytes, proliferation of synovial cells and extensive angiogenesis, which is collectively referred to as the rheumatoid pannus [2-4]. A critical phenomenon occurring in the early stages of synovial inflammation is angiogenesis [4,5], which commences with the activation of endothelial cells by a variety of stimuli, including pro-inflammatory cytokines and growth factors such as vascular endothelial growth factor (VEGF). The affected endothelial cells (ECs) then begin to digest the basement membrane, proliferate, migrate and eventually differentiate to form a tubular structure [6].

Fas (also known as CD95) was initially discovered as a cell surface molecule that efficiently triggers death signals when bound to its ligand, Fas ligand (FasL) [7,8]. Fas is ubiquitously expressed, whereas FasL is principally expressed on activated T cells [7], natural killer cells [9], tumor cells [10], and in immune privileged sites such as the eye [11]. The Fas-FasL interaction plays a pivotal role in activation-induced cell death of T lymphocytes [12], and is responsible for the cytotoxicity of T lymphocytes [13,14] and natural killer cells [9]. Consequently, Fas and FasL are crucial components of lymphocyte homeostasis. In addition to the homeostatic regulation of the immune system, Fas and FasL are involved in tumor

$\overline{\mathrm{CD} 40 \mathrm{~L}}=\mathrm{CD} 40$ ligand; DMEM = Dulbecco's modified Eagle's medium; EC = endothelial cell; ELISA = enzyme-linked immunosorbent assay; FCS $=$ fetal calf serum; FasL = Fas ligand; FLS = fibroblast-like synoviocyte; Flt = fms-like tyrosine kinase; ITSA = insulin-transferrin-selenium A; KDR = kinase insert domain-containing receptor; $\mathrm{mFasL}=$ membrane-associated FasL; OA = osteoarthritis; $\mathrm{pAkt}=\mathrm{phospho-Akt;} \mathrm{PBS=phosphate-buffered}$ saline; $\mathrm{pERK}=$ phospho-extracellular signal-regulated kinase; RA = rheumatoid arthritis; sFasL $=$ soluble FasL; TGF = transforming growth factor; TNF = tumor necrosis factor; VEGF = vascular endothelial growth factor. 
surveillance $[15,16]$. Moreover, Fas and FasL are thought to inhibit angiogenesis by inducing apoptosis of either ECs or leukocytes that provide angiogenic growth factors [17-20], although one study reported an increase in angiogenesis by Fas and FasL [21]. Similar to tumor necrosis factor (TNF)- $\alpha$, FasL is cleaved from the cell surface by a metalloproteinase [22]. The released form of FasL, soluble FasL (sFasL), was originally thought to induce apoptosis in a manner similar to membrane-associated FasL (mFasL) [23]. However, there have been many subsequent reports upholding the differences between $s F a s L$ and $m F a s L$ regarding apoptosis induction $[24,25]$. Despite the numerous studies on the role of Fas and FasL in immune homeostasis, the effect of sFasL on the angiogenic process of RA remains to be determined.

In this study, we tested whether sFasL can regulate angiogenesis and apoptosis of rheumatoid synoviocytes. We demonstrate here that sFasL potently decreased VEGF $_{165}$ production by RA fibroblast-like synoviocytes (FLSs) by inducing apoptosis in vitro. In addition, sFasL effectively inhibited VEGF $_{165}$-induced migration and chemotaxis of ECs, although it did not affect tube formation by ECs. The effect of sFasL on ECs was not due to Fas-mediated cell death, since sFasL did not change either spontaneous or $\mathrm{VEGF}_{165}$-stimulated EC proliferation or survival. Moreover, sFasL strongly inhibited neovascularization in the Matrigel plug in vivo. Taken together, sFasL inhibits angiogenesis within RA synovium not only by inducing apoptosis of VEGF ${ }_{165}$-producing cells such as FLSs, but also by blocking $\mathrm{VEGF}_{165}$-induced migration of ECs, independent of Fas-mediated apoptosis.

\section{Materials and methods Culture of RA synoviocytes and collection of synovial fluids}

The RA FLSs were prepared from the synovial tissues of ten RA patients that were undergoing total joint replacement surgery, as described previously [26]. The mean age of the RA patients ( 9 females and 1 male) was 48.3 years. Eight patients had a positive rheumatoid factor. Osteoarthritis (OA) FLSs, isolated from 5 female OA patients (mean age 66.2 years), were used as a control. Synovial tissues were minced into 2 to $3 \mathrm{~mm}$ pieces, and treated for 4 hours with $4 \mathrm{mg} / \mathrm{ml}$ of type I collagenase (Worthington Biochemical, Freehold, NJ, USA) in DMEM at $37^{\circ} \mathrm{C}$ in a $5 \% \mathrm{CO}_{2}$ atmosphere. Dissociated cells were then resuspended in DMEM, supplemented with $10 \%$ FCS, $2 \mathrm{mM}$ glutamine, penicillin and streptomycin, and then plated in $75 \mathrm{~cm}^{2}$ flasks. After overnight culturing, the nonadherent cells were removed and adherent cells cultivated in DMEM plus $10 \%$ FCS. Cultures were kept at $37^{\circ} \mathrm{C}$ in a $5 \%$ $\mathrm{CO}_{2}$ atmosphere, and the medium was replaced every 3 days. At confluence, the cells were passed by diluting $1: 3$ with fresh medium and re-cultured until used. Synoviocytes, from passages 4 through 8 , were used for each experiment. The FLSs were washed in DMEM and then incubated for an additional 24 hours in serum-free DMEM supplemented with insulin- transferrin-selenium A (ITSA; Life Technologies, Grand Island, NY, USA). The cells $\left(3 \times 10^{4}\right.$ cells/well) were seeded in triplicate into 24-well plates (Nunc, Roskilde, Denmark) in serumfree DMEM (supplemented with ITSA) without or with transforming growth factor (TGF)- $\beta$ (PeproTech, London, UK) in the presence of sFasL (10 to $100 \mathrm{ng} / \mathrm{ml}$; MBL, Nagoya, Japan). After the indicated hours of incubation, cell viability was tested by 3-(4,5-dimethylthiazol-2-yl)-2,5-diphenyltetrazolium bromide (MTT) assay, and the cell-free media were collected to measure VEGF ${ }_{165}$ concentration in the culture supernatants. Synovial fluid was obtained with informed consent from RA and $\mathrm{OA}$ patients with joint effusion, and stored at $-20^{\circ} \mathrm{C}$ in a refrigerator. All samples were obtained according to the guidelines approved by the Ethics Committee of the Catholic University of Korea.

\section{ELISA for $\mathbf{S F a s}$ and VEGF $\mathbf{1}_{\mathbf{1 6 5}}$}

The amount of sFasL and VEGF VE5 $_{165}$ was measured by ELISA, as previously described [27]. Recombinant human sFasL and $\operatorname{VEGF}_{165}$ (R \& D, Minneapolis, MN, USA) were used as a calibration standard. A standard curve was drawn by plotting the optical density versus the log of the concentration of sFas and VEGF $_{165}$.

\section{Isolation and culture of endothelial cells}

The ECs were isolated from normal-term umbilical cord vein by collagenase digestion, and then grown to confluence in 75 $\mathrm{cm}^{2}$ flasks containing M199 medium (Life Technologies) supplemented with $20 \% \mathrm{FCS}, 100 \mathrm{U} / \mathrm{ml}$ penicillin, $100 \mu \mathrm{g} / \mathrm{ml}$ streptomycin, and $2 \mathrm{mM} \mathrm{L-glutamine.} \mathrm{Cultures} \mathrm{were} \mathrm{kept} \mathrm{at}$ $37^{\circ} \mathrm{C}$ in a $\mathrm{CO}_{2}$ incubator, and the medium was changed every 2 to 3 days until confluence was reached. ECs were passed with $0.2 \%$ collagenase and $0.02 \%$ EDTA (Life Technologies); cells from passages 2 to 3 were used in this study.

\section{Determination of endothelial cell proliferation and viability}

The proliferation and survival of ECs were assessed by $\left[{ }^{3} \mathrm{H}\right]-$ thymidine incorporation assay and MTT assay, respectively. Briefly, ECs were incubated for 24 hours in DMEM supplemented with $1 \%$ FCS. The medium was replaced with fresh DMEM/ITSA supplemented with $1 \%$ FCS, and the cells were incubated without or with $10 \mathrm{ng} / \mathrm{ml}$ of $\mathrm{VEGF}_{165}$ in the presence of sFasL ( 1 to $100 \mathrm{ng} / \mathrm{ml}$ ) for 24 hours. For the determination of the proliferation rate of ECs, $1 \mu \mathrm{Ci}$ of $\left[{ }^{3} \mathrm{H}\right]$-thymidine was added to each of the wells prior to the final 6 hours of culturing, and the incorporated radioactivity was counted with a scintillation counter.

\section{Wounding migration and tube formation assay}

The wounding migration and tube formation activity of ECs were measured as described previously [28]. In brief, ECs plated at confluence on $60 \mathrm{~mm}$ culture dishes were wounded with pipette tips, and then treated with $\operatorname{VEGF}_{165}(20 \mathrm{ng} / \mathrm{ml})$ in M199 medium, supplemented with 1\% FCS and $1 \mathrm{mM}$ of 
thymidine. After 12 hours of incubation, migration was quantified by counting the cells that had moved beyond the reference line. For the tube formation assay, ECs were seeded on a layer of previously polymerized Matrigel (BD Biosciences, San Jose, CA, USA) with VEGF ${ }_{165}(20 \mathrm{ng} / \mathrm{ml})$. After 18 hours of incubation, the cell morphology was visualized via phasecontrast microscopy and photographed. The degree of tube formation was quantified by measuring the length of tubes in 5 randomly chosen low-power fields $(\times 40)$ from each well using image-Pro Plus v4.5 (Media Cybernetics, San Diego, CA, USA).

\section{Chemotaxis assay of endothelial cells}

The chemotactic migration of ECs was assayed using a Transwell chamber with $6.5 \mathrm{~mm}$ diameter polycarbonate filters (8 $\mu \mathrm{m}$ pore size). In brief, the filter's lower surface was coated with $10 \mu \mathrm{g}$ of gelatin. VEGF ${ }_{165}(10 \mathrm{ng} / \mathrm{ml})$, which was prepared in M199 medium containing 1\% FCS, was placed in the lower wells. The ECs that were incubated in M199 with 1\% FCS for 6 hours or overnight were trypsinized and suspended at a final concentration of $1 \times 10^{6} \mathrm{cells} / \mathrm{ml}$ in M199 containing $1 \%$ FCS. Various concentrations of sFasL or soluble CD40 ligand (sCD40L; R \& D) were added to the upper wells with $100 \mu \mathrm{l}$ of cell suspensions. The chamber was incubated at $37^{\circ} \mathrm{C}$ for 4 hours. The cells were fixed and stained with hematoxylin and eosin. Non-migrating cells on the filter's upper surface were removed by wiping with a cotton swab. Chemotaxis was quantified by counting the cells that migrated to the lower side of the filter by optical microscopy at $\times 200$ magnification. Eight random fields were counted for each assay. Each sample was assayed in duplicate.

\section{Western blot analysis for phospho-Akt and phospho- ERK}

ECs were incubated for 24 hours in DMEM with $1 \%$ FCS, and then $\mathrm{VEGF}_{165}(20 \mathrm{ng} / \mathrm{ml})$ plus various concentrations of sFasL ( 1 to $50 \mathrm{ng} / \mathrm{ml}$ ) were added to the cells for the indicated times. The treated ECs were then washed twice in PBS, dissolved in sample buffer (50 mM Tris- $\mathrm{HCl}, 100 \mathrm{mM} \mathrm{NaCl}, 0.1 \%$ SDS, $1 \% \mathrm{NP}-40,50 \mathrm{mM} \mathrm{NaF}, 1 \mathrm{mM} \mathrm{Na} \mathrm{VO}_{4}, 1 \mu \mathrm{g} / \mathrm{ml}$ aprotinin, 1 $\mu \mathrm{g} / \mathrm{ml}$ pepstatin, and $1 \mu \mathrm{g} / \mathrm{ml}$ leupeptin), boiled, separated via SDS-PAGE, and transferred to nitrocellulose membranes. After immunoblot analysis with anti-phospho-ERK1/2 (Thr202/Tyr204) or anti-phospho-Akt (Ser473), the membranes were stripped and re-incubated with $\beta$-actin antibody in order to detect total protein amounts.

\section{Mouse Matrigel plug assay}

Matrigel $(500 \mu \mathrm{l})$ containing VEGF ${ }_{165}(500 \mathrm{ng} / \mathrm{ml})$ and heparin (9 U/ml) were injected subcutaneously with or without sFasL $(100 \mathrm{ng} / \mathrm{ml})$ into the abdomen of C57BL/6 mice (7 weeks of age), as described previously [28]. After 14 days, the skins of the mice were pulled back to expose the Matrigel plugs, which remained intact. After noting and photographing any quantitative differences, hemoglobin levels were measured by the
Figure 1

(a)

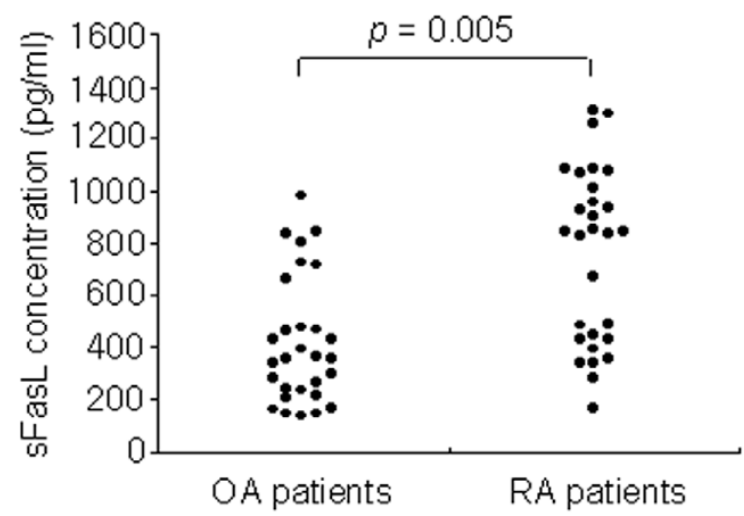

(b)

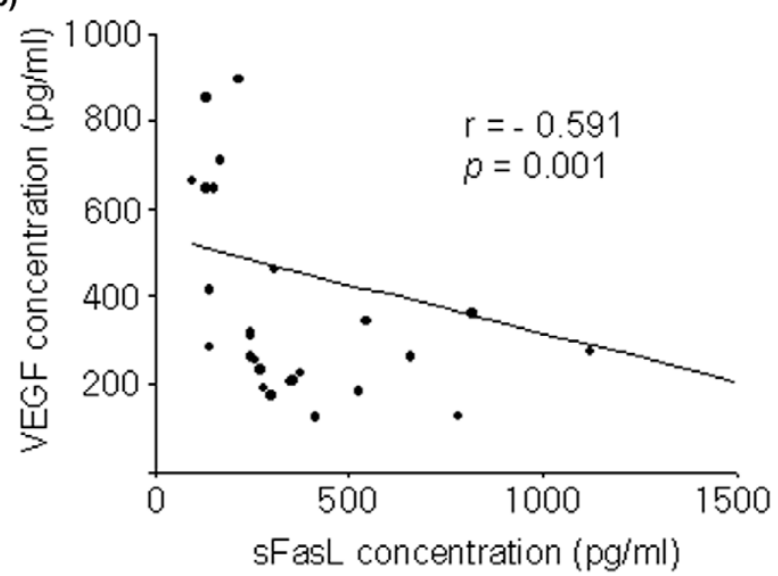

(c)

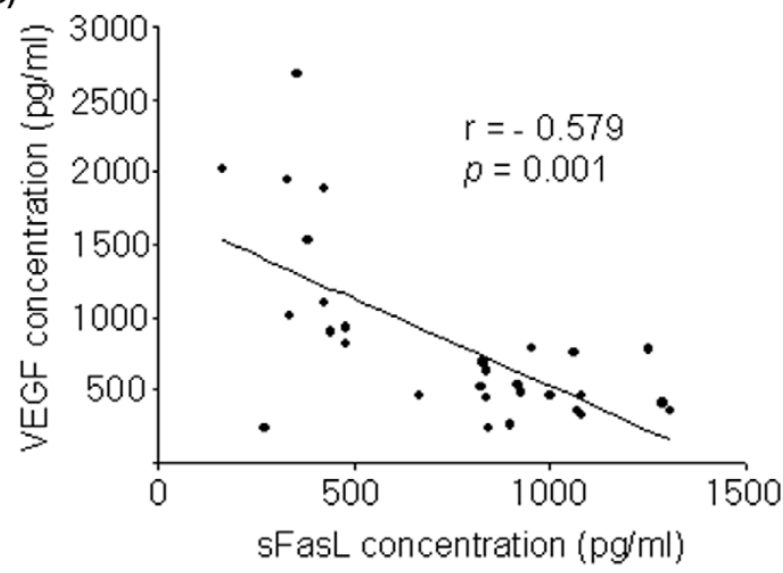

Inverse correlation between soluble Fas ligand (sFasL) and vascular endothelial growth factor (VEGF) levels in patients with rheumatoid arthritis (RA). (a) Concentrations of sFasL in synovial fluid of patients with RA $(n=29)$ and osteoarthritis $(\mathrm{OA}, n=30)$. (b) Correlation of VEGF $_{165}$ concentration with sFasL level in the sera of patients with RA. (c) Correlation between $\mathrm{VEGF}_{165}$ and sFasL levels in the synovial fluid of patients with RA. 
Figure 2

(a)

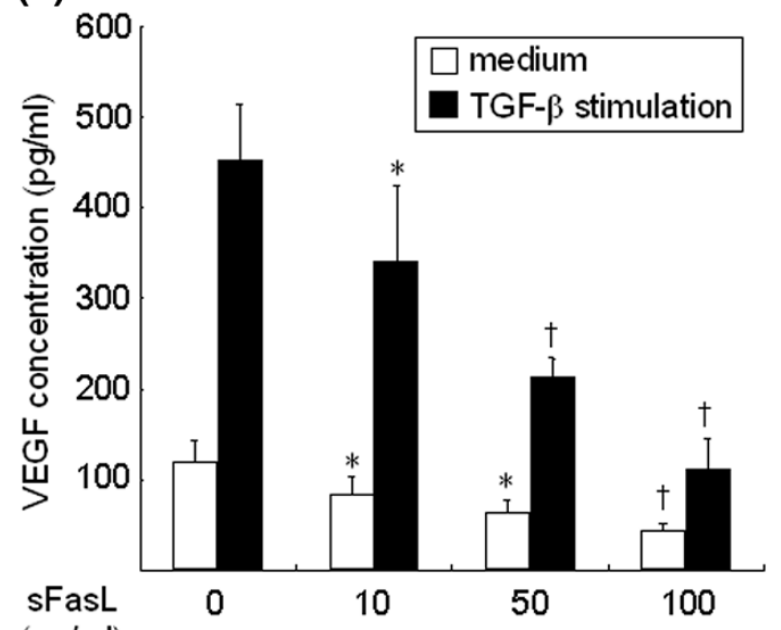

(b)

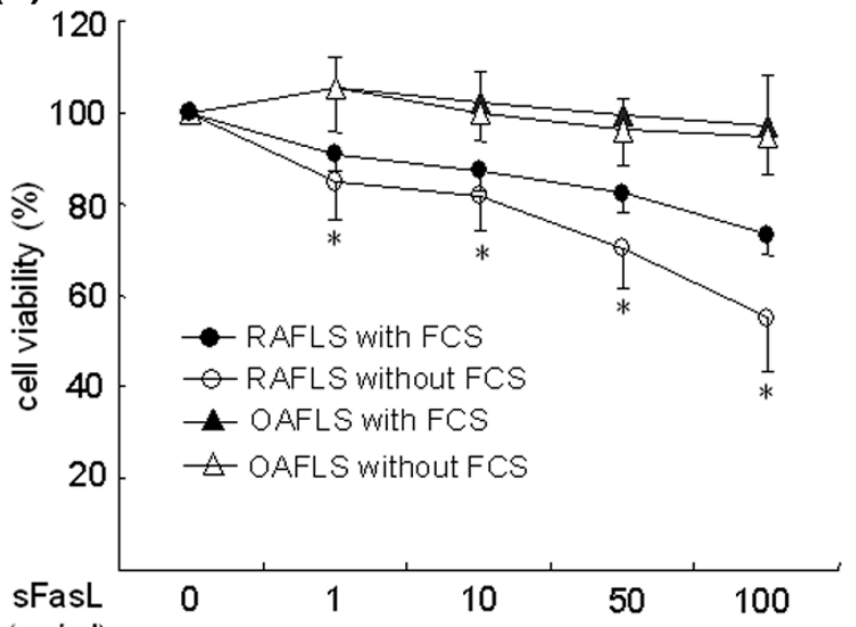

$(\mathrm{ng} / \mathrm{ml})$

$(\mathrm{ng} / \mathrm{ml})$

Effect of soluble Fas ligand (sFasL) on vascular endothelial growth factor (VEGF) ${ }_{165}$ production by synovial fibroblasts. (a) Fibroblast-like synoviocytes (FLSs) were cultured in triplicate for 24 hours with medium alone and transforming growth factor (TGF)- $\beta$ ( $10 \mathrm{ng} / \mathrm{ml})$ in the presence of various concentrations of sFasL (10 to $100 \mathrm{ng} / \mathrm{ml})$. The amount of $\mathrm{VEGF}_{165}$ in the culture supernatants was determined by ELISA. Data are the mean \pm standard deviation (SD) of three independent experiments in triplicate. ${ }^{\star} P<0.05 ;{ }^{+} P<0.01$ versus the cells stimulated with medium alone or TGF$\beta$ in the absence of sFasL. (b) The rheumatoid arthritis FLSs (RAFLS; $n=7$ ) or osteoarthritis FLSs (OAFLS; $n=5$ ) were treated with increasing concentrations of sFasL ( 1 to $100 \mathrm{ng} / \mathrm{ml}$ ) in the absence or presence of $1 \%$ FCS for 24 hours. The viability of FLS was determined by the 3-(4,5-dimethylthiazol-2-yl)-2,5-diphenyltetrazolium bromide (MTT) assay. Data are expressed as the mean \pm SD. * $P<0.01$ versus OAFLS.

Drabkin method, using a Drabkin reagent kit 525 (Sigma, St. Louis, MO, USA). The hemoglobin concentration was calculated from the parallel assay of a known amount of hemoglobin. The Matrigel plugs were fixed in $4 \%$ formalin, embedded with paraffin, and stained using hematoxylin and eosin.

\section{Statistical analysis}

Data are expressed as the mean \pm standard deviation (SD). Comparisons of the numerical data between groups were performed by paired or unpaired Mann-Whitney U-test. $P$ values less than 0.05 are considered statistically significant.

\section{Results}

\section{sFasL and VEGF $_{165}$ levels correlate inversely in patients} with RA

Using ELISA, sFasL was detected in synovial fluids from RA patients $(n=29)$ and OA patients $(n=30)$ (Figure 1a). As with another reported study [27], the concentration of sFasL in the synovial fluid of RA patients was significantly higher than that of OA patients (mean levels of sFasL: $753 \pm 335$ versus 474 $\pm 401 \mathrm{pg} / \mathrm{ml}, P=0.005)$. It has been reported that there is an inverse correlation between apoptotic (FasL, caspase-3) and angiogenic (VEGF, microvessel density) factors in squamous cell lung carcinomas [19]. To determine the relationship between sFasL and VEGF ${ }_{165}$, the serum and synovial fluids of RA patients were simultaneously measured for sFasL and VEGF $_{165}$ by ELISA. As reported earlier [29], the VEGF ${ }_{165}$ level was significantly higher in synovial fluids of RA patients than those of OA patients (mean levels of VEGF VE5 $_{162}: 823615$ versus $230 \pm 131 \mathrm{pg} / \mathrm{ml}, P=0.015)$. In RA patients, there were strong negative correlations between VEGF $_{165}$ and sFasL levels in the sera $(r=-0.591, P=0.001)$ and synovial fluids $(r=$ -0.579, $P=0.001$ ) (Figure 1b,c). However, sFasL levels in OA synovial fluids did not show any correlation with VEGF ${ }_{165}$ concentrations (data not shown).

\section{SFasL decreases VEGF $_{165}$ production by synovial fibroblasts via apoptosis induction}

It is well known that FLSs are the major site of VEGF ${ }_{165}$ production in RA joints [30]. Based on the finding of an inverse correlation between sFasL and VEGF ${ }_{165}$ levels in RA synovial fluids, we attempted to determine the effects of sFasL on VEGF $_{165}$ production by FLSs in vitro. For this, RA FLSs were cultured in the presence of sFasL for 24 hours either with medium alone or with the supplementary addition of TGF- $\beta$ (10 ng/ml), since TGF- $\beta$ has been described to strongly induce VEGF ${ }_{165}$ [31,32]. As seen in Figure 2a, sFasL, ranging from 10 to $100 \mathrm{ng} / \mathrm{ml}$, dose-dependently decreased both spontaneous and TGF- $\beta$-stimulated VEGF $_{165}$ production by RA FLSs cultured without FCS. The sFasL-induced decrease in VEGF $_{165}$ production appears to be mediated by cell death, because similar concentrations of sFasL decreased FLS survival to $55 \%$ of the basal level, as determined by MTT assay (Figure 2b). The sFasL-mediated cell death was partially blocked by co-treating RA FLSs $(n=7)$ with $1 \%$ FCS (Figure $2 b)$. OA FLSs $(n=5)$ were resistant to sFasL-induced cell 
Figure 3

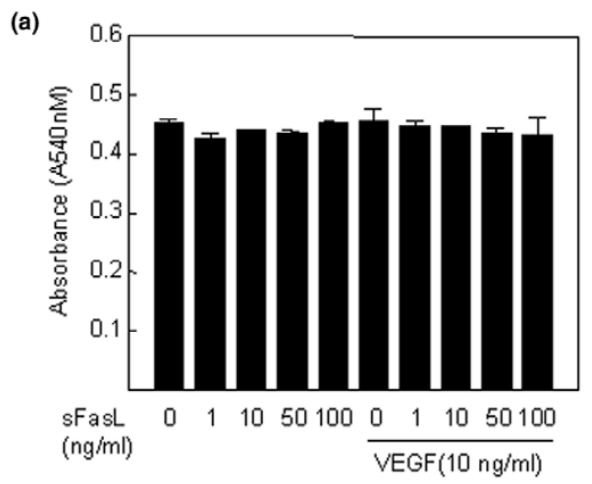

(b)

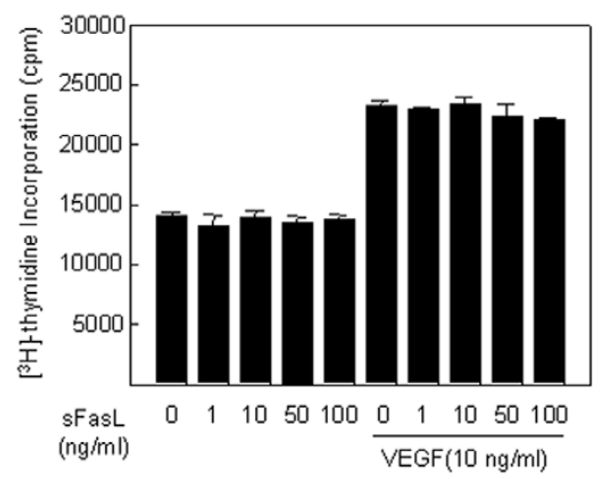

(c)

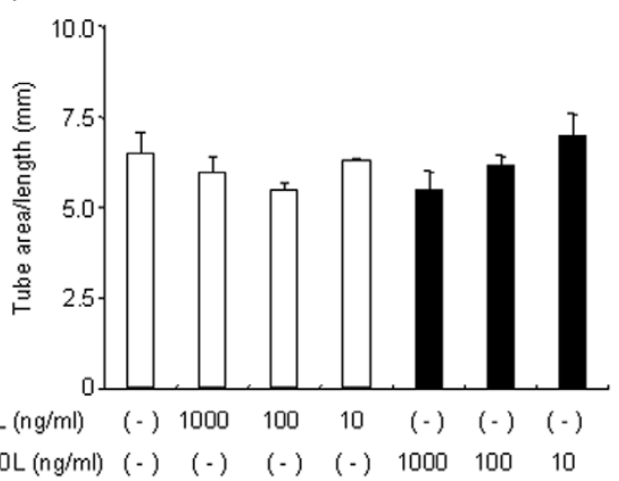

Soluble Fas ligand (sFasL) does not affect the survival, proliferation and tube formation of endothelial cells (ECs). $(a, b)$ No effect of sFasL on the survival (a) and proliferation (b) of ECs. The ECs were incubated for 24 hours in DMEM supplemented with 1\% FCS, and were treated with various concentrations of sFasL ( 1 to $100 \mathrm{ng} / \mathrm{ml}$ ) in the absence or presence of vascular endothelial growth factor (VEGF) ${ }_{165}(10 \mathrm{ng} / \mathrm{ml})$. The viability and proliferation of ECs were determined by the 3-(4,5dimethylthiazol-2-yl)-2,5-diphenyltetrazolium bromide (MTT) and $\left[{ }^{3} \mathrm{H}\right]-$ thymidine incorporation assays, respectively. Data are expressed as the mean \pm standard deviation (SD) of three independent experiments. (c) No effect of sFasL on tube formation by ECs. The ECs were plated on Matrigel matrices with VEGF ${ }_{165}(20 \mathrm{ng} / \mathrm{ml})$ in the presence of various concentrations (10 to $1,000 \mathrm{ng} / \mathrm{ml}$ ) of sFasL or soluble CD40 ligand (sCD40L) for 48 hours. The total length of the tube network was calculated using Image-Pro Plus software. Data are representative of three independent experiments with similar results, presented as the mean \pm SD. death, indicating that the apoptotic action of sFasL may be specific to RA FLSs. These results, together with the data presented in Figure 1, suggest that sFasL may indirectly participate in anti-angiogenesis by eliminating $\mathrm{VEGF}_{165}$-producing cells in RA joints, but not in OA joints.

\section{Effect of sFasL on angiogenesis of ECs in vitro}

To investigate the effect of sFasL on the survival and proliferation of ECs, these cells were cultured with various concentrations of sFasL ( 1 to $100 \mathrm{ng} / \mathrm{ml}$ ) in the absence or presence of $1 \%$ FCS. As expected, EC death was induced dose-dependently by treating the cells with sFasL in the absence of FCS (data not shown). However, when 1\% FCS was added to the EC culture, sFasL failed to affect EC survival and proliferation, which were assessed by MTT assay and $\left[{ }^{3} \mathrm{H}\right]$-thymidine incorporation assay, respectively (Figure $3 a, b$ ). The VEGF VE5- $^{-}$ induced formation of tube-like structures by ECs was also not altered by the addition of sFasL (10 to $100 \mathrm{ng} / \mathrm{ml}$; Figure 3c). In angiogenesis, ECs migrate in response to several chemotactic factors [6]. Therefore, we attempted to elucidate whether sFasL affects $\mathrm{VEGF}_{165}$-induced EC migration and chemotaxis. As shown in Figure $4 \mathrm{a}$, sFasL suppressed VEGF $_{165}$-induced wounding migration of ECs. Moreover, sFasL strongly inhibited VEGF ${ }_{165}$-induced chemotaxis of ECs, determined by using a Transwell chamber (Figure 4b), whereas SCD40L $(0.1$ to $100 \mathrm{ng} / \mathrm{ml})$ did not affect it. These data suggest that sFasL inhibits angiogenesis by blocking migration and chemotaxis of ECs, independent of Fas-mediated cell death.

\section{sFasL inhibits VEGF $_{\mathbf{1 6 5}^{-}}$-induced upregulation of phospho-Akt expression}

VEGF $_{165}$ exerts its biological effects by binding to its receptors, which include fms-like tyrosine kinase (Flt-1) and kinase insert domain-containing receptor (KDR) [33]. Flt-1 and KDR exhibit tyrosine kinase activity, and both are expressed in the majority of ECs $[33,34]$. KDR is a primary mediator of EC proliferation in response to $\mathrm{VEGF}_{165}$. Unlike KDR, Flt-1 is present in inflammatory cells, and mediates chemotaxis of ECs $[33,34]$. Thus, we sought to investigate whether sFasL regulates the expression of VEGF receptors or their signaling pathways in ECs. Our results show that sFasL failed to inhibit the mRNA and protein expression of Flt and KDR expression, as determined by reverse transcription PCR and Western blot analysis, respectively (data not shown). However, the VEGF $_{165}$-induced expression of phospho-Akt (pAkt), one of the downstream targets for Flt signaling [35], was dosedependently inhibited with sFasL treatment, whereas phospho-extracellular signal-regulated kinase (pERK) activity, which is critical for EC proliferation [35], remained unchanged (Figure 5). Taken together, these data suggest that sFasL may inhibit migration and chemotaxis of ECs by blocking VEGF ${ }_{165^{-}}$ induced upregulation of pAkt activity. 
Figure 4

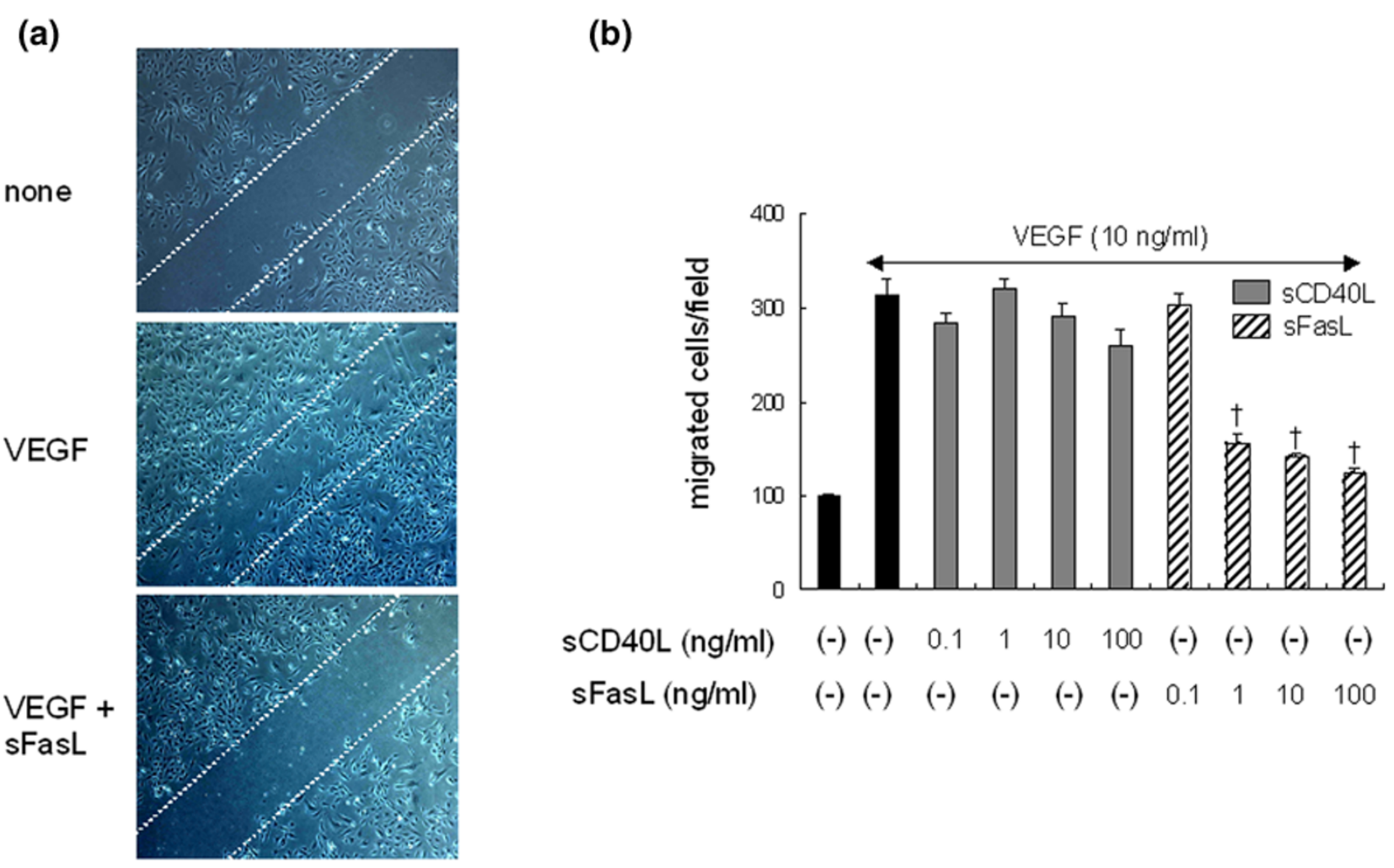

Soluble Fas ligand (sFasL) strongly inhibits migration and chemotaxis of endothelial cells (ECs). (a) Inhibition of wounding migration of ECs by sFasL. Confluent ECs were wounded with the tip of a micropipette, and incubated further in M199 containing 1\% FCS with vascular endothelial growth factor (VEGF) ${ }_{165}(20 \mathrm{ng} / \mathrm{ml})$ in the absence or presence of sFasL $(50 \mathrm{ng} / \mathrm{ml})$. After 12 hours, the cells migrating beyond the reference line were photographed (at $\times 50$ magnification) and counted. A representative of three independent experiments is shown. (b) sFasL suppresses the chemotaxis of ECs induced by VEGF ${ }_{165}$. The ECs were placed in the upper wells of the chemotaxis chamber with various concentrations $(0.1$ to $100 \mathrm{ng} / \mathrm{ml}$ ) of soluble CD40 ligand (sCD4OL) or sFasL in the presence of VEGF (10 ng/ml) added in the lower wells. The number of cells that had migrated to the lower surface of the membrane was counted after hematoxylin and eosin staining. Data are representative of three independent experiments, presented as the mean \pm standard deviation. ${ }^{\dagger} P<0.01$ versus the $\mathrm{VEGF}_{165}$-stimulated cells without sFasL.

\section{SFasL inhibits angiogenesis in vivo}

We finally attempted to determine whether sFasL blocks VEGF $_{165}$-induced neovascularization in vivo. The in vivo exposed Matrigel mixtures containing $\mathrm{VEGF}_{165}(500 \mathrm{ng} / \mathrm{ml})$ were colored orange to red, whereas the gels containing $V_{E G F}{ }_{165}$ plus sFasL $(100 \mathrm{ng} / \mathrm{ml})$ retained their original white to amber coloring (Figure 6a). In an attempt to quantify the angiogenesis in these samples, we measured the hemoglobin contents of the Matrigel mixture gels. The mean hemoglobin content of the VEGF VE5 $_{16}$-treated Matrigels $(n=8)$ was $8.30 \pm$ $3.52 \mathrm{~g} / \mathrm{dl}$, whereas that of the $\mathrm{VEGF}_{165}$ plus sFasL-treated Matrigels $(n=8)$ was $1.20 \pm 0.26 \mathrm{~g} / \mathrm{dl}(P<0.001)$ (Figure $6 b)$. The stained sections indicate that Matrigels containing $\mathrm{VEGF}_{165}$ had produced more vessels in the gels than had the Matrigel containing the $\mathrm{VEGF}_{165}$ plus sFasL (Figure $6 \mathrm{c}$ ). These new vessels were filled with an abundance of intact red blood cells, indicating the formation of a functional vasculature within the Matrigels. These results appear to suggest that sFasL has potent anti-angiogenic activity in vivo as well as in vitro.

\section{Discussion}

FasL is a type II membrane protein of approximately 280 amino acids that belongs to the TNF/nerve growth factor family, which includes TNF, lymphotoxin, CD4OL, and TRAIL. FasL induces apoptotic death in cells expressing its receptor, Fas $[17,35]$. FasL undergoes proteolytic cleavage in its extracellular domains, resulting in the release of sFasL [22]. In general, sFasL is less potent at inducing apoptosis than membranebound Fas, as shown in a variety of cell types $[24,25,36]$. Elevated levels of $s F a s L$ are found in sera from patients with atherosclerosis [37], leukemia [38], and acute graft-versus host disease [39]. The concentration of sFasL in the synovial fluid is higher in patients with severe RA than in those with mild RA or OA [27]. Moreover, local injection of sFasL into the affected joints suppresses experimental arthritis in rats [40], suggesting it has therapeutic potential in RA.

Angiogenesis has been considered a critical step in the progression of chronic arthritis, as well as an early determinant in the development of RA [41]. In this study, we first identified a 
Figure 5
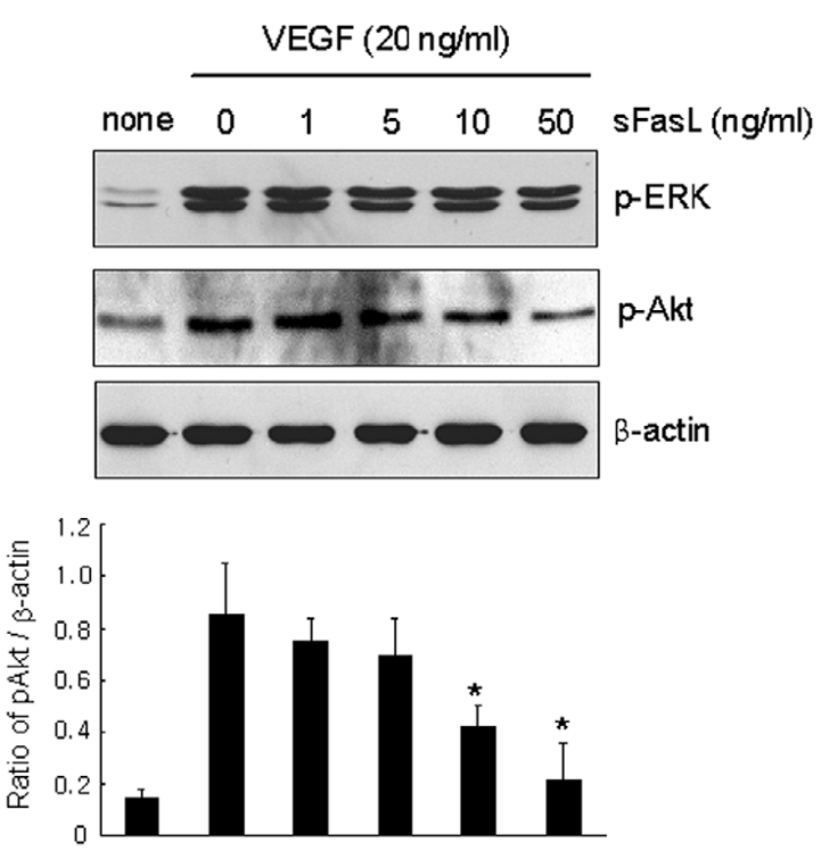

Inhibition of vascular endothelial growth factor (VEGF) 165 -induced phsospho-Akt (pAkt) activity by soluble Fas ligand (sFasL). Endothelial cells (ECs) were incubated with various concentrations of sFasL ( 1 to $50 \mathrm{ng} / \mathrm{ml})$ in the presence of VEGF ${ }_{165}(20 \mathrm{ng} / \mathrm{ml})$ for 10 minutes. The pAkt and phospho-extracellular signal-regulated kinase (pERK) expression levels in the ECs were determined by western blot analysis. The data are a representative result of three independent experiments, and are expressed as the mean ( \pm standard deviation) optical density ratio [pAkt/ $\beta$-actin]. ${ }^{*} P<0.05$ versus the $\mathrm{VEGF}_{165}$-stimulated cells without sFasL.

novel mechanism for anti-angiogenesis in RA involving sFasL. sFasL decreased VEGF ${ }_{165}$ production from RA FLSs by inducing apoptosis in vitro. The apoptotic action of sFasL seems to be specific to RA FLSs because it minimally affected the viability of OA FLSs, which is consistent with the previous finding that OA FLSs are less sensitive to Fas-mediated apoptosis [42]. In addition, sFasL drastically suppressed VEGF $_{165}{ }^{-}$ induced migration and chemotaxis of ECs in vitro and also blocked neovascularization in vivo, although it did not alter the proliferation and tube formation of ECs, indicating that sFasL displays anti-angiogenic activity through at least two different mechanisms: induction of Fas-mediated cell death of VEGF $_{165}$-producing cells; and apoptosis-independent inhibition of EC migration and chemotaxis. These data, together with earlier reports $[15,40]$, suggest that administration of sFasL could be effective in treating several angiogenesisdependent diseases, such as cancer and chronic inflammatory diseases, explaining how sFasL protects against the development of experimental arthritis.

RA FLSs are susceptible to anti-Fas IgM and undergo apoptosis in vitro [43,44]. By contrast, apoptotic cells are rarely observed in the RA synovium in vivo [43]. The effect of FasL
Figure 6

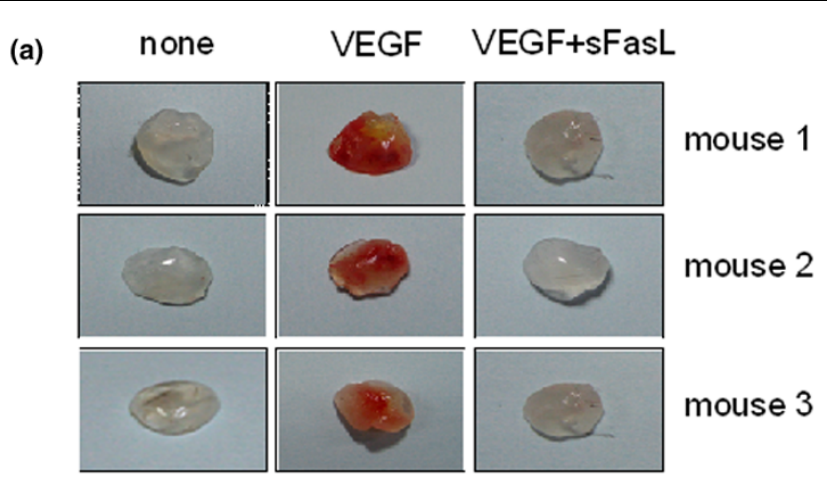

(b)

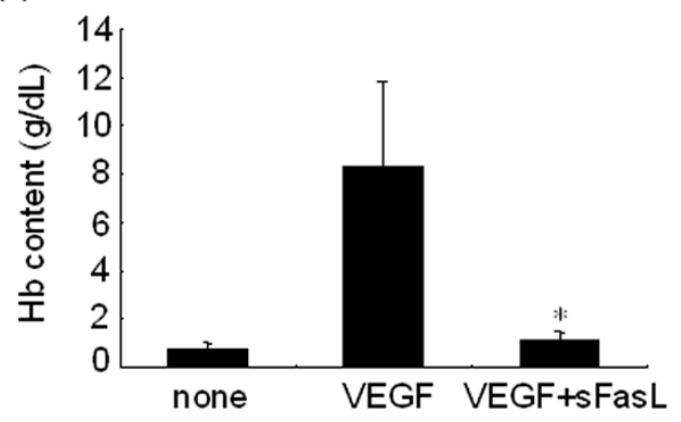

(c) VEGF VEGF+sFasL

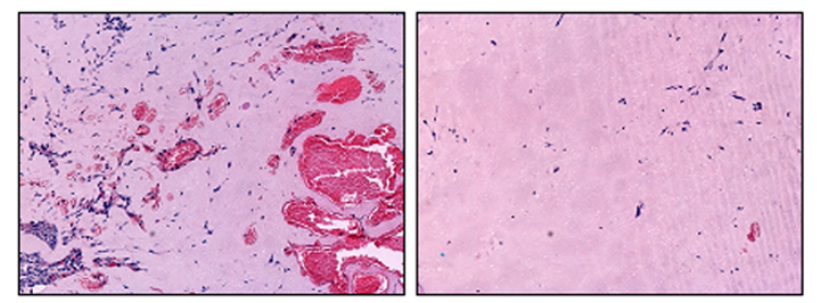

Effect of soluble Fas ligand (sFasL) on neovascularization in vivo. Matrigel containing vascular endothelial growth factor (VEGF) 165 and heparin were injected subcutaneously with or without sFasL (100 ng/ $\mathrm{ml}$ ) into the abdomen of C57BL/6 mice. After 14 days, the mice were sacrificed and the Matrigel plugs were excised and fixed. (a) Representative Matrigel plugs containing PBS (none), VEGF ${ }_{165}(500 \mathrm{ng} / \mathrm{ml})$, or VEGF ${ }_{165}(500 \mathrm{ng} / \mathrm{ml})$ plus sFasL $(100 \mathrm{ng} / \mathrm{ml})$. (b) Quantification of new vessel formation via measurements of the hemoglobin $(\mathrm{Hb})$ content within the Matrigel is shown. Eight mice were used for each group. Data represent the mean \pm standard deviation, and similar results were obtained with two different experiments. ${ }^{*} P<0.001$ versus the $\mathrm{Hb}$ content of the Matrigel containing VEGF ${ }_{165}$. (c) Representative photograph of the gels shown in cross-section and stained with hematoxylin and eosin. Original magnification $(\times 100)$.

on apoptosis of ECs is also still controversial; although some studies report that ECs are sensitive to FasL-induced apoptosis $[45,46]$, others report the contrary $[47,48]$. In our experiments, sFasL was able to induce the apoptosis of RA FLSs and ECs in a culture condition without FCS (Figure 2b; data 
not shown). However, when 1\% FCS was added to the medium, sFasL-mediated cell death was partially inhibited in the cultured FLSs or did not occur in ECs (Figures $2 b$ and $3 a$ ), indicating that the apoptotic action of sFasL on VEGF-secreting cells, such as FLSs, was hampered by growth factors. Recently, we have demonstrated that VEGF ${ }_{165}$ protects FLSs from apoptotic death by regulating $\mathrm{Bcl}-2$ expression and $\mathrm{Bax}$ translocation [49]. Therefore, it seems unlikely that sFasL greatly elicits apoptosis of FLSs and ECs in the joints with high levels of VEGF VE5 $_{165}$ or other growth factors. Instead, a mechanism by which sFasL induces anti-angiogenesis by blocking migration and chemotaxis of ECs may be more relevant in this specific condition.

Fas ligation activates a pro-inflammatory program in some cell types independently of apoptotic responses [50-52]. For example, Fas stimulation increased expression of $\mathrm{I} \kappa \mathrm{B} \alpha$, matrix metalloproteinases and chemokines, and Fas-activated RA FLSs displayed increased chemotactic activity for monocytic cells [50]. In our study, Fas ligation by sFasL did not affect the production of chemokines, such as IL-8 and monocyte chemoattractant protein-1, by ECs (data not shown), suggesting that Fas ligation does not trigger chemotactic signals for ECs. On the contrary, sFasL treatment blocked the migration and chemotaxis of ECs induced by VEGF ${ }_{165}$ (Figure 4). Moreover, sFasL strongly inhibited VEGF ${ }_{165}$-induced pAkt activity, but not pERK activity, in ECs (Figure 5). It is unclear how sFasL regulates $V_{E G F}$-induced pAkt activity and then suppresses the chemotaxis and migration of ECs. Activation of the phosphatidylinositol 3-kinase/protein kinase Akt pathway mediates nitric oxide-induced EC migration and angiogenesis [53]. Conversely, inhibition of the Akt pathway results in anti-angiogenic effects through the inhibition of EC migration in an apoptosis-independent manner [54,55]. Given that the activities of Flt-1 and its downstream target pAkt are critical to VEGF $_{165}$ signaling for chemotaxis [53,54], the anti-migratory action of sFasL may be mediated by blocking VEGF ${ }_{165^{-}}$ induced upregulation of pAkt activity. Further study is required to determine the molecular mechanisms for sFasL regulation of pAkt activity linked to chemotaxis control.

\section{Conclusion}

We demonstrate here that FasL showed anti-angiogenic activity not only by inducing apoptosis of VEGF-producing cells but also by blocking VEGF-induced migration of ECs, independent of Fas-mediated apoptosis. Our data suggest that SFasL may be effective in the treatment of RA, and could be applicable to the modulation of various chronic VEGF-dependent inflammatory diseases.

\section{Competing interests}

The authors declare that they have no competing interests.

\section{Authors' contributions}

WK participated in the design of the study, drafted the manuscript and analyzed the data. SK helped to draft the manuscript. KH carried out cell cultures and ELISA, and helped to perform migration, chemotaxis, and tube formation assays of ECs. JK and SU assisted in cell cultures and performed migration, chemotaxis, and tube formation assays of ECs, and the in vivo Matrigel assay. JC participated in the design of the study and the analysis of data. CC conceived of the study, participated in its design and coordination, and helped to draft the manuscript. All authors read and approved the final manuscript.

\section{Acknowledgements}

This work was supported by grant no. R01-2006-000-11359-0 from the Basic Research Program of the Korea Science and Engineering Foundation and Second-Phase of Brain Korea 21. We express our appreciation to $\mathrm{Dr}$ Mi-Sook Lee for help with some experiments on angiogenesis.

\section{References}

1. Feldmann M, Brennan FM, Maini RN: Rheumatoid arthritis. Cell 1996, 85:307-310.

2. Firestein GS: Invasive fibroblast-like synoviocytes in rheumatoid arthritis. Passive responders or transformed aggressors? Arthritis Rheum 1996, 39:1781-1790.

3. Reece RJ, Canete JD, Parsons WJ, Emery P, Veale DJ: Distinct vascular patterns of early synovitis in psoriatic, reactive, and rheumatoid arthritis. Arthritis Rheum 1999, 42:1481-1484.

4. Koch $\mathrm{AE}$ : Angiogenesis as a target in rheumatoid arthritis. Ann Rheum Dis 2003, 62(Suppl 2):ii60-67.

5. Fearon U, Griosios K, Fraser A, Reece R, Emery P, Jones PF, Veale DJ: Angiopoietins, growth factors, and vascular morphology in early arthritis. J Rheumato/ 2003, 30:260-268.

6. Risau W: Mechanisms of angiogenesis. Nature 1997, 386:671-674.

7. Suda T, Takahashi T, Golstein P, Nagata S: Molecular cloning and expression of the Fas ligand, a novel member of the tumor necrosis factor family. Cell 1993, 75:1169-1178.

8. Owen-Schaub LB, Yonehara S, Crump WL 3rd, Grimm EA: DNA fragmentation and cell death is selectively triggered in activated human lymphocytes by Fas antigen engagement. Cell Immunol 1992, 140:197-205.

9. Oshimi Y, Oda S, Honda Y, Nagata S, Miyazaki S: Involvement of Fas ligand and Fas-mediated pathway in the cytotoxicity of human natural killer cells. J Immunol 1996, 157:2909-2915.

10. Strand S, Hofmann WJ, Hug H, Muller M, Otto G, Strand D, Mariani SM, Stremmel W, Krammer PH, Galle PR: Lymphocyte apoptosis induced by CD95 (APO-1/Fas) ligand-expressing tumor cells - a mechanism of immune evasion? Nat Med 1996, 2:1361-1366.

11. Griffith TS, Brunner T, Fletcher SM, Green DR, Ferguson TA: Fas ligand-induced apoptosis as a mechanism of immune privilege. Science 1995, 270:1189-1192.

12. Ju ST, Panka DJ, Cui H, Ettinger R, el-Khatib M, Sherr DH, Stanger BZ, Marshak-Rothstein A: Fas(CD95)/FasL interactions required for programmed cell death after T-cell activation. Nature 1995, 373:444-448.

13. Hanabuchi S, Koyanagi M, Kawasaki A, Shinohara N, Matsuzawa A, Nishimura Y, Kobayashi Y, Yonehara S, Yagita H, Okumura K: Fas and its ligand in a general mechanism of T-cell-mediated cytotoxicity. Proc Natl Acad Sci USA 1994, 91:4930-4934.

14. Lowin B, Hahne M, Mattmann C, Tschopp J: Cytolytic T-cell cytotoxicity is mediated through perforin and Fas lytic pathways. Nature 1994, 370:650-652.

15. Wajant $\mathrm{H}$ : CD95L/FasL and TRAIL in tumour surveillance and cancer therapy. Cancer Treat Res 2006, 130:141-165.

16. Davidson WF, Giese T, Fredrickson TN: Spontaneous development of plasmacytoid tumors in mice with defective Fas-Fas ligand interactions. J Exp Med 1998, 187:1825-1838. 
17. Lee HO, Ferguson TA: Biology of FasL. Cytokine Growth Factor Rev 2003, 14:325-335.

18. Kaplan HJ, Leibole MA, Tezel T, Ferguson TA: Fas ligand (CD95 ligand) controls angiogenesis beneath the retina. Nat Med 1999, 5:292-297.

19. Volm M, Mattern J, Koomagi R: Inverse correlation between apoptotic (Fas ligand, caspase-3) and angiogenic factors (VEGF, microvessel density) in squamous cell lung carcinomas. Anticancer Res 1999, 19:1669-1671.

20. Panka DJ, Mier JW: Canstatin inhibits Akt activation and induces Fas-dependent apoptosis in endothelial cells. J Biol Chem 2003, 278:37632-37636.

21. Biancone L, Martino AD, Orlandi V, Conaldi PG, Toniolo A, Camussi G: Development of inflammatory angiogenesis by local stimulation of Fas in vivo. J Exp Med 1997, 186:147-152.

22. Kayagaki N, Kawasaki A, Ebata T, Ohmoto H, lkeda S, Inoue S, Yoshino K, Okumura K, Yagita H: Metalloproteinase-mediated release of human Fas ligand. J Exp Med 1995, 182:1777-1783.

23. Tanaka M, Suda T, Takahashi T, Nagata S: Expression of the functional soluble form of human fas ligand in activated lymphocytes. EMBO J 1995, 14:1129-1135.

24. Tanaka M, Itai T, Adachi M, Nagata S: Downregulation of Fas ligand by shedding. Nat Med 1998, 4:31-36.

25. Schneider $\mathrm{P}$, Holler N, Bodmer JL, Hahne M, Frei K, Fontana A, Tschopp J: Conversion of membrane-bound Fas(CD95) ligand to its soluble form is associated with downregulation of its proapoptotic activity and loss of liver toxicity. J Exp Med 1998, 187:1205-1213.

26. Yoo SA, Park BH, Park GS, Koh HS, Lee MS, Ryu SH, Miyazawa $\mathrm{K}$, Park SH, Cho CS, Kim WU: Calcineurin is expressed and plays a critical role in inflammatory arthritis. J Immunol 2006, 177:2681-2690.

27. Hashimoto H, Tanaka M, Suda T, Tomita T, Hayashida K, Takeuchi E, Kaneko M, Takano H, Nagata S, Ochi T: Soluble Fas ligand in the joints of patients with rheumatoid arthritis and osteoarthritis. Arthritis Rheum 1998, 41:657-662.

28. Lee MS, Moon EJ, Lee SW, Kim MS, Kim KW, Kim YJ: Angiogenic activity of pyruvic acid in in vivo and in vitro angiogenesis models. Cancer Res 2001, 61:3290-3293.

29. Lee SS, Joo YS, Kim WU, Min DJ, Min JK, Park SH, Cho CS, Kim HY: Vascular endothelial growth factor levels in the serum and synovial fluid of patients with rheumatoid arthritis. Clin Exp Rheumatol 2001, 19:321-324.

30. Fava RA, Olsen NJ, Spencer-Green G, Yeo KT, Yeo TK, Berse B, Jackman RW, Senger DR, Dvorak HF, Brown LF: Vascular permeability factor/endothelial growth factor (VPF/VEGF): accumulation and expression in human synovial fluids and rheumatoid synovial tissue. J Exp Med 1994, 180:341-346.

31. Berse B, Hunt JA, Diegel RJ, Morganelli P, Yeo K, Brown F, Fava RA: Hypoxia augments cytokine (transforming growth factorbeta (TGF-beta) and IL-1)-induced vascular endothelial growth factor secretion by human synovial fibroblasts. Clin Exp Immunol 1999, 115:176-182.

32. Pertovaara L, Kaipainen A, Mustonen T, Orpana A, Ferrara N, Saksela $\mathrm{O}$, Alitalo $\mathrm{K}$ : Vascular endothelial growth factor is induced in response to transforming growth factor-beta in fibroblastic and epithelial cells. J Biol Chem 1994, 269:6271-6274.

33. Ferrara N, Gerber HP, LeCouter J: The biology of VEGF and its receptors. Nat med 2003, 9:669-676.

34. Autiero M, Luttun A, Tjwa M, Carmeliet P: Placental growth factor and its receptor, vascular endothelial growth factor receptor1: novel targets for stimulation of ischemic tissue revascularization and inhibition of angiogenic and inflammatory disorders. J Thromb Haemost 2003, 1:1356-1370.

35. Kowanetz M, Ferrara N: Vascular endothelial growth factor signaling pathways: therapeutic perspective. Clin Cancer Res 2006, 12:5018-5022.

36. Sieg S, Smith D, Kaplan D: Differential activity of soluble versus cellular Fas ligand: regulation by an accessory molecule. Cell Immunol 1999, 195:89-95.

37. Okura $T$, Watanabe $S$, Jiang $Y$, Nakamura $M$, Takata $Y$, Yang $Z$, Kohara K, Kitami Y, Hiwada K: Soluble Fas ligand and atherosclerosis in hypertensive patients. J Hypertens 2002, 20:895-898

38. Saitoh T, Karasawa M, Sakuraya M, Norio N, Junko T, Shirakawa K, Matsushima T, Tsukamoto N, Nojima Y, Murakami H: Improvement of extrathymic $\mathrm{T}$ cell type of large granular lymphocyte
(LGL) leukemia by cyclosporin A: the serum level of Fas ligand is a marker of LGL leukemia activity. Eur J Haematol 2000, 65:272-275

39. Kanda Y, Tanaka Y, Shirakawa K, Yatomi T, Nakamura N, Kami M, Saito T, Izutsu K, Asai T, Yuji K, et al:: Increased soluble Fas-ligand in sera of bone marrow transplant recipients with acute graft-versus-host disease. Bone Marrow Transplant 1998, 22:751-754.

40. Li NL, Nie H, Yu OW, Zhang JY, Ma AL, Shen BH, Wang L, Bai J, Chen $\mathrm{XH}$, Zhou T, Zhang DQ: Role of soluble Fas ligand in autoimmune diseases. World J Gastroenterol 2004, 10:3151-3156.

41. Koch $A E$ : Review: angiogenesis: implications for rheumatoid arthritis. Arthritis Rheum 1998, 41:951-962.

42. Okamoto K, Fujisawa K, Hasunuma T, Kobata T, Sumida T, Nishioka K: Selective activation of the JNK/AP-1 pathway in Fasmediated apoptosis of rheumatoid arthritis synoviocytes. Arthritis Rheum 1997, 40:919-926.

43. Peng SL: Fas (CD95)-related apoptosis and rheumatoid arthritis. Rheumatology (Oxford) 2006, 45:26-30.

44. Baier A, Meineckel I, Gay S, Pap T: Apoptosis in rheumatoid arthritis. Curr Opin Rheumatol 2003, 15:274-279.

45. Filippatos G, Ang E, Gidea C, Dincer E, Wang R, Uhal BD: Fas induces apoptosis in human coronary artery endothelial cells in vitro. BMC Cell Biol 2004, 5:6.

46. Janin A, Deschaumes C, Daneshpouy M, Estaquier J, Micic-Polianski J, Rajagopalan-Levasseur P, Akarid K, Mounier N, Gluckman E Socie G, Ameisen JC: CD95 engagement induces disseminated endothelial cell apoptosis in vivo: immunopathologic implications. Blood 2002, 99:2940-2947.

47. Mogi M, Fukuo K, Yang J, Suhara T, Ogihara T: Hypoxia stimulates release of the soluble form of fas ligand that inhibits endothelial cell apoptosis. Lab Invest 2001, 81:177-184.

48. Sata M, Suhara T, Walsh K: Vascular endothelial cells and smooth muscle cells differ in expression of Fas and Fas ligand and in sensitivity to Fas ligand-induced cell death: implications for vascular disease and therapy. Arterioscler Thromb Vasc Biol 2000, 20:309-316.

49. Kim WU, Kang SS, Yoo SA, Hong KH, Bae DG, Lee MS, Hong SW, Chae CB, Cho CS: Interaction of vascular endothelial growth factor ${ }_{165}$ with neuropilin-1 protects rheumatoid synoviocytes from apoptotic death by regulating bcl-2 expression and bax translocation. J Immunol 2006, 177:5727-5735.

50. Palao G, Santiago B, Galindo MA, Rullas JN, Alcami J, Ramirez JC, Pablos JL: Fas activation of a proinflammatory program in rheumatoid synoviocytes and its regulation by FLIP and caspase 8 signaling. Arthritis Rheum 2006, 54:1473-1481.

51. Choi C, Xu X, Oh JW, Lee SJ, Gillespie GY, Park H, Jo H, Benveniste EN: Fas-induced expression of chemokines in human glioma cells: involvement of extracellular signal-regulated kinase $1 / 2$ and p38 mitogen-activated protein kinase. Cancer Res 2001, 61:3084-3091.

52. Ahn JH, Park SM, Cho HS, Lee MS, Yoon JB, Vilcek J, Lee TH: Non-apoptotic signaling pathways activated by soluble Fas ligand in serum-starved human fibroblasts. Mitogen-activated protein kinases and NF-kappaB-dependent gene expression. $J$ Biol Chem 2001, 276:47100-47106.

53. Kawasaki K, Smith RS Jr, Hsieh CM, Sun J, Chao J, Liao JK: Activation of the phosphatidylinositol 3-kinase/protein kinase Akt pathway mediates nitric oxide-induced endothelial cell migration and angiogenesis. Mol Cell Biol 2003, 23:5726-5737.

54. Dell'Eva R, Ambrosini C, Minghelli S, Noonan DM, Albini A, Ferrari $\mathrm{N}$ : The Akt inhibitor deguelin, is an angiopreventive agent also acting on the NF-KB pathway. Carcinogenesis 2007, 28:404-413.

55. Murtagh J, Lu H, Schwartz EL: Taxotere-induced inhibition of human endothelial cell migration is a result of heat shock protein 90 degradation. Cancer Res 2006, 66:8192-8199. 